\title{
Vehicle Classification Based on Locational Matrix and Edge Region Minimization
}

\author{
Mingfang Zhang ${ }^{1, \mathrm{a}}$, $\mathrm{Li} \mathrm{Li}^{1, \mathrm{~b}}$ and Yu Cui ${ }^{1, \mathrm{C}}$ \\ ${ }^{1}$ School of Automobile, Chang'an University, Xi'an, China \\ a957631035@qq.com, b991958405@qq.com, c1121406285@qq.com
}

\begin{abstract}
Keywords: Locational matrix; edge; classification; grid partition; similarity probability
Abstract. Due to the influence of gray contrast ratio on background image with the target vehicle, traditional vehicle detection algorithms cannot adapt to complicated traffic environment. This paper proposes a new vehicle classification method based on locational matrix and minimum of local edge region. First, the lane line is determined by gray gradient variation and the regions surrounded by lane lines is regarded as the recognition region for further vehicle detection. Second, grid partition of the original image is conducted and classification label of each grid is determined by the assumed gray threshold. Then locational matrix is obtained based on the label number of the corresponding grids and the similarity rate is analyzed with different $\varepsilon$ to get the optimal $\varepsilon$ value. The relationship between the distance from the mean of local region gray to the connected domain of vehicle body and $\varepsilon$ is the iteration termination condition. Finally, vehicle outline edge is refined through the minimum of edge local region. Test results show that our method classifies vehicle outline edge accurately, compared with single image match method.
\end{abstract}

\section{Introduction}

Vehicle image system provides the technical support for assistant driving which has become a inevitable part of intelligent vehicle. The efficiency of vehicle detection determines the intelligence level of assistant driving system. However, the complexity of the traffic scene directly restricts the wide application of image processing technology in the field of intelligent vehicle. Yusuke Kanzawa proposed the low resolution image flow frame construction to obtain the image features of the medium distance vehicles to solve the low resolution of vehicle image in the long range, achieving the vehicle tracking and detection [1]. The image stream is acquired through the vehicle camera, and the image is processed by the observation of multiple low resolution images, and the image is reconstructed with high resolution image. Arrospide Jon considers the orientation gradient histogram (HOGs) is the most effective feature in vehicle image, but the HOGs calculation cost is too large and difficult to detect the vehicle [2]. Zhou Chenyu transforms support vector machine of the image feature detection into two classification problem of the background image and the target image [3]. The vehicle detection algorithms depend on the gray level change of the original image, and it is difficult to meet the practical requirements. In order to solve this problem, the paper proposes to construct the locational feature matrix and detects the vehicle with the edge gray level characteristics under complex environment. The algorithm greatly reduces the dependence on the image gray gradient and has a simple calculation process.

\section{Image locational matrix}

Image filtering. Improving the quality of the car camera can provide a wide range of applications for the vehicle auxiliary system [4]. Affected by illumination changes, vehicle body vibration, road shadow and dust in the air, many noise points exist on the vehicle image through the vehicle camera. In order to enhance the contrast between the target vehicle and the background image, the original image is required to filter. We select Canny operator to process the original image [5]. The processing results are shown in Fig. 2. 


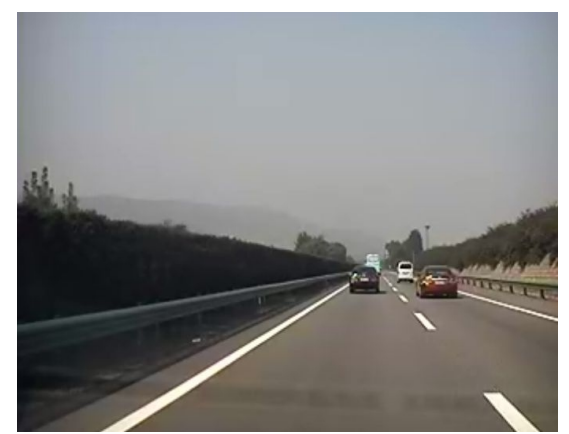

Fig.1 Original image

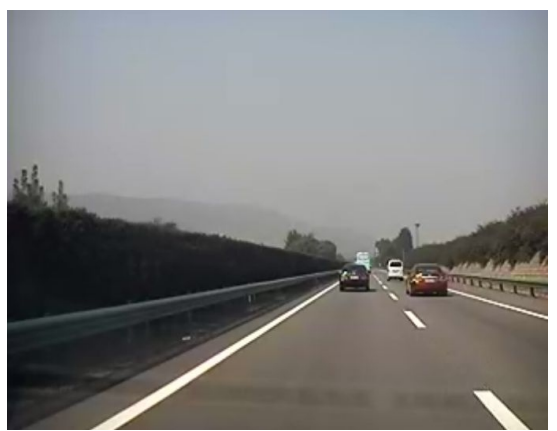

Fig.2 Filtered image

After the image is filtered, the gray value at the edge of each connected region is enhanced. The scene in Figure 1 shows many vehicles are distant from the test vehicle, and the contrast between the measured object edge and background is not obvious. If the whole panorama is searched, the computation is large and the segmentation is not accurate. Since the contrast between lane marking line and exterior scene is significant, and the test vehicle is located in the enclosed area blocked by lane mark line. Therefore, the contour of the lane mark line is extracted, the area to be searched is initially determined. Because the lane marking line is continuous, the outer edge of the lane can be determined by continuous decision.

Grid partition. In order to ensure the consistency of flow size for the same exterior scene, mesh of the original image. The large grid number will increase the searching complexity, and the small grid number will affect to the original feature of the target, so the grid number should be small to keep target edge feature clear. In order to determine the appropriate number of grid, the number of grid is determined by the detection algorithm accuracy rate according to different number of grid and the restriction of search time. The detection accuracy rate is highest when the grid number is $16 * 16$.

Image locational matrix confirmation. The gray value of all pixels in a single grid is used as the matrix element, and the original image size is $\mathrm{N}^{*} \mathrm{M}$, then the pixel number of each element in the locational matrix $\mathrm{T}$ is,

$$
\mathrm{C}_{\text {number }}=(N / 16)(M / 16) \text {. }
$$

In order to ensure the consistency of the pixels in each grid, the original image is complemented with $T_{0}(i, 1), T_{0}(i, 16), T_{0}(1, j), T_{0}(16, j)$. The label of each grid should be determined with the certain grid number, and the gray level value of each grid can be classified into two categories with the assumed gray threshold. The gray value of each grid $S_{i j}$ is

$$
S_{i j}=\frac{\sum_{k_{1}=1}^{[n / 16]} \sum_{k_{2}=1}^{[M / 16]} T_{0}\left((i-1)[N / 16]+k_{1},(j-1)[M / 16]+k_{2}\right)}{[N / 16][M / 16]} .
$$

Where $\mathrm{i}$, j represents the row and column element of image matrix. Assumed gray threshold is $S_{0}$, if the following conditions are satisfied, a single grid label can be set to 1 , otherwise it is 0 ,

$$
L_{i j}= \begin{cases}1 & \mathrm{~S}_{i j} \geq S_{0} \\ 0 & \text { otherwise. }\end{cases}
$$

The locational matrix is obtained by calculating the grid number which is labeled as 1 in the corresponding rows and columns. The final locational matrix $\mathrm{L}_{0}$ is

$$
L_{0}=\left[\begin{array}{llllllllllllllll}
0 & 0 & 0 & 0 & 7 & 4 & 6 & 5 & 5 & 1 & 3 & 0 & 0 & 0 & 0 & 0 \\
0 & 0 & 0 & 0 & 0 & 0 & 1 & 5 & 6 & 5 & 5 & 3 & 4 & 2 & 0 & 0
\end{array}\right]^{T}
$$


Edge statistical analysis. The vehicle contour is obtained with locational matrix, and the contour edge of the vehicle is further refined based on the rough contour. The gray scale of the rough edges in the local area show double peak characteristics.

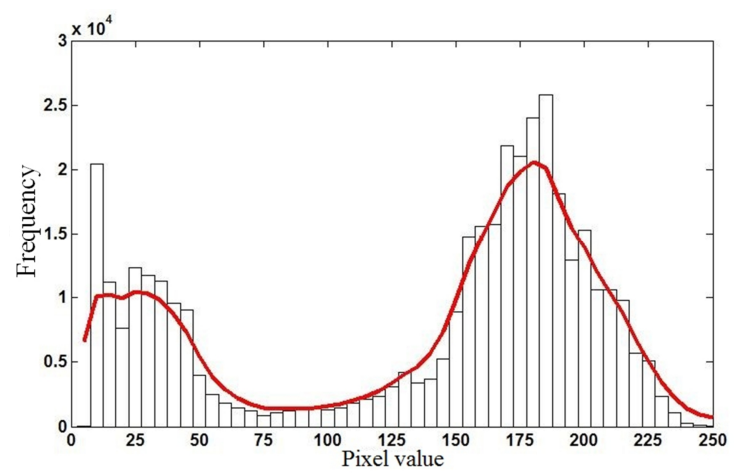

Fig.3 Gray statistics graph of part edge region

From Fig.3, the double peak characteristics of gray level for the edge of the local area is obvious. With the assumed gray threshold of the body region, the pixels in the local area are reduced, and the local area with the edge is minimized, then the clear coutour edge is obtained. Set the initial value for the local width of the edge as $N$ (i.e., the pixels containing in local area width), the mean value of gray level in vehicle body region is $S_{v}$, the mean of gray level in the background region is $S_{b}$, the total number of pixels in the edge local region is $N_{0}$. Thus, gray value of the edge local area $G_{0}$ is $\frac{\sum_{i=1}^{N} G(i)}{N}$, $G(i)$ is the gray value. The search process is terminated if the following conditions is satisfied.

$$
\left|\frac{G_{k}-S_{v}}{G_{k}-S_{b}}\right|=\left|\frac{\sum_{i=1}^{N_{i}} G(i)}{N_{i}}-S_{v}\right| \leq \varepsilon .
$$

Where $\varepsilon$ is constant and it is less than $1, G_{k}$ is the mean of gray level in k edge local area. If the distance between gray mean in local region and vehicle body region is less than the mean value of the background gray, the searching will terminate. $\varepsilon$ is related to the iteration number and the edge detection accuracy. Small $\varepsilon$ makes the edge subdivision unclear and big $\varepsilon$ makes the iteration number increasing and affects the convergence. Therefore, it is crucial to determine the appropriate $\varepsilon$. We select the appropriate $\varepsilon$ based on the ratio of the pixels in vehicle region to the pixels in real vehicle region with different $\varepsilon$, as shown in Fig.4. Balancing iteration number with detection similarity probability, finally we set $\varepsilon$ as 0.3 .

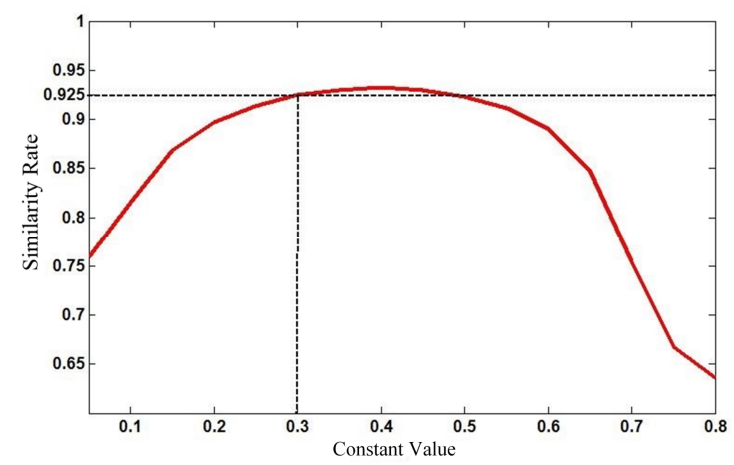

Fig.4 Detection similarity probability change along with $\varepsilon$ 
Vehicle classification. Our proposed vehicle recognition algorithm mainly includes establishing vehicle locational matrix and edge refining. For collected panoramas, the search region surrounded by the lane mark line is obtained by image denoising, enhancement and lane line extraction. When determining the locational matrix, the exterior search area is set as 0 and it means no vehicle exists in that region. Finally, the edge of the vehicle contour is refined with the minimization of edge local region for the region determined by the locational matrix, and then the target vehicle is identified. Fig. 5 and Fig. 6 are respectively the test result from single image matching and vehicle edge determined by the minimization of edge local.

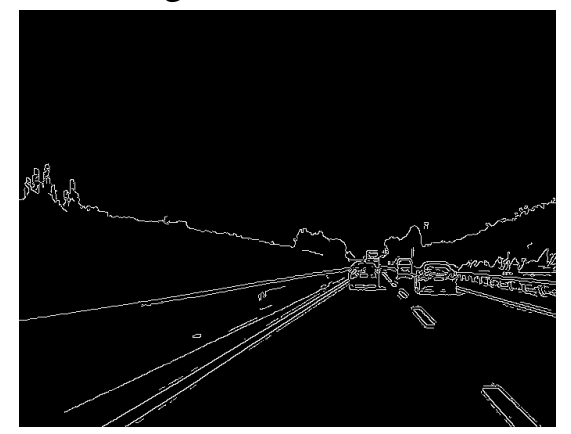

Fig.5 Result of single image matching method

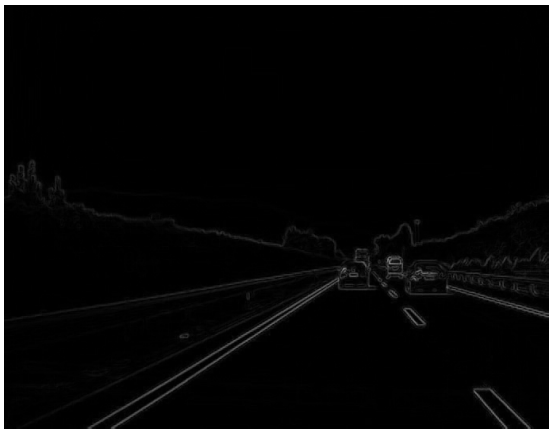

Fig.6 Result of our method

From the above figures, clear lane marking line is obtained by single image matching, but the detection result is obviously not good for multiple detection targets, our proposed method not only can accurately detect lane marking line, but it can get clearer contour edge. And computing time is less than single image matching method while the detection efficiency is more than $94 \%$, even in complex traffic environment.

\section{Conclusion}

The traditional image matching technology is heavily dependent on gray scale, which affects the efficiency of vehicle detection algorithm with video system on the vehicles. In order to recognize the vehicles, our proposed method first exploits the locational matrix and edge local characteristics. Then image denoising and enhancement are conducted with the raw image and grid partition is realized for the panorama. Further, the lane mark line is extracted based on the contrast between the lane mark line and the background, and grid labels are compared to construct the locational matrix. Finally the vehicle contour edge is refined based on the minimization of edge local region. Experimental result shows the algorithm in this paper is more efficient than the traditional single image matching method. It has low computational complexity and can realize multiple vehicle recognition in complex scene.

\section{References}

[1] Y. Kanzawa, H. Kobayashi. Low-resolution Vehicle Image Recognition Technology by Frame Composition of Moving Image. 2010, 93(3):1-7.

[2] J. Arrospide, L. Salgado, M. Camplani. Image-based on-road Vehicle Detection Using Cost-effective Histograms of Oriented Gradients. Journal of Visual Communication and Image Representation, 2013, 24(7):100-108.

[3] C.Y. ZHOU, Y.Q. ZHANG. Recognition Technology of Vehicle Based on SVM. Science \& Techonlogy Review, 2012, 33(30):53-57. In Chinese.

[4] W. Liu, X. Wen, B. Duan. Rear Vehicle Detection and Tracking for Lane Change Assist. Proceedings of the 2007 IEEE Intelligent Vehicles Symposium, Istanbul, Turkey, 2007:13-15.

[5] S. CHEN, Y.Q. HOU, X.L. YANG. Mixed Denoising Method Based on Partial Differential Equation and Wiener Filtering. Computer Engineering, 2010, 36(10):193-195. In Chinese. 